\title{
Sudden singularities survive massive quantum particle production
}

\author{
John D. Barrow, ${ }^{1, *}$ Antônio B. Batista, ${ }^{2, \dagger}$ Júlio C. Fabris, ${ }^{2, \ddagger}$ Mahouton J. S. Houndjo, ${ }^{3, \S}$ and Giuseppe Dito ${ }^{4,5, \|}$ \\ ${ }^{1}$ DAMTP, Centre for Mathematical Sciences, University of Cambridge, UK \\ ${ }^{2}$ Departamento de Física, Universidade Federal do Espírito Santo, ES, Brazil \\ ${ }^{3}$ Instituto de Física, Universidade Federal da Bahia, Ba, Brazil \\ ${ }^{4}$ Instituto de Matemática, Universidade Federal da Bahia, Ba, Brazil \\ ${ }^{5}$ Institut de Mathématiques de Bourgogne, Université de Bourgogne, Dijon, France
}

(Received 7 October 2011; published 23 December 2011)

\begin{abstract}
We solve the Klein-Gordon equation for a massive, nonminimally coupled scalar field, with a conformal coupling, undergoing cosmological evolution from a radiation-dominated phase to a future sudden singularity. We show that, after regularization, the energy of the created particles is zero and the backreaction from quantum effects does not change the evolution of the Universe near the future singularity and cannot prevent the finite-time sudden singularity.
\end{abstract}

DOI: 10.1103/PhysRevD.84.123518

PACS numbers: $98.80 .-\mathrm{k}$

\section{INTRODUCTION}

The combined data from the anisotropy of the cosmic microwave background radiation, supernovae type Ia, baryonic acoustic oscillations and matter power spectrum indicate that the dominant component of the mass-energy content of the universe must be gravitationally repulsive and driving the acceleration of the universal expansion. In general, such a component can be represented by a fluid with an equation of state $p=\omega \rho$, with $\omega<-\frac{1}{3}$. If $\omega$ is constant and the spatial sections of the Universe is flat, the recent results of seven years of WMAP observations indicate that $\omega=-1.10 \pm 0.14$ [1] at $1 \sigma$. This indicates that the null energy condition is violated, and the expanding Universe may hit a universal singularity in its future evolution, after a finite proper time. This type of future singularity has been named "big rip," when there is a divergence in the density of the exotic dark energy fluid, while the scale factor goes to infinity [2]. This strange behavior is connected with the violation of the null energy condition. The possibility that the null energy condition $(\rho+p \geq 0)$ is violated, and cosmological singularities may occur in the future evolution of the Universe, has been evoked frequently in the literature in the last years. The possibility of future singularities was pointed out for the first time in Ref. [3] and their occurrence does not require the violation of the energy conditions. There is also a later discussion in Ref. [4]. A recent example of future singularities which does not violate the null energy condition is the "big brake" singularity, which emerges from the DBI action $[5,6]$. The big brake singularity has the curious property that it can be traversed by a pointlike particle [7].

\footnotetext{
*jdb34@damtp.cam.ac.uk

abrasilb918@gmail

†abrisjc@yahoo.com.br

${ }^{\S}$ sthoundjo@yahoo.fr. Present address: ICRA-CBPF, RJ, Brazil

" giuseppe.dito@u-bourgogne.fr
}

A milder type of finite-time singularity is the so-called "sudden singularity." The sudden future singularity occurs without violation of any energy condition (so $\rho+p \geq 0$ and $\rho+3 p \geq 0$ at all times). This singularity is characterized by a finite value for the scale factor, its first time derivative, and for the density, while the second derivative of the scale factor and the pressure diverge at finite time [3]. They are singularities of the weak sort discussed by Tipler [8] and Krolak [9].

In general, it is believed that the fate of the universe near a singularity (past or future) must be affected by quantum effects arising in the extreme conditions that exist in its spacetime neighborhood. In the case of the big rip, this problem has been treated, for example, in Refs. [10-12]. In these investigations, it was found that the quantum effects are important. But, the conclusions concerning the backreaction of the quantum effects on the evolution of the Universe were harder to decide unambiguously. In the case of the sudden singularity, quantum effects were studied in Refs. $[13,14]$, and the results indicated that quantum effects did not change the evolution near the singularity. These results were all obtained for a massless scalar field.

It is important to extend these results to the case of massive scalar fields. Here, we extend earlier studies to the case of a massive scalar field, nonminimally coupled to gravity, with a conformal coupling parameter, in a universe which possesses a sudden singularity when quantum effects are absent. We will show that the singularity is unscathed by the backreaction from the quantum field. The complexity of the background evolution of the sudden singularity restricts the possibility of a complete analytical solution. Hence, we simplify the model by considering two phases: a primordial radiative phase and a sudden singular phase. Under these conditions, we can determine the evolution of the quantum fields. In particular, we can calculate the Bogoliubov coefficients and determine the energy density of the particles created by quantum effects near the singularity. After regularization, we find a null result 
implying that the sudden singularity persists, unaffected, by the quantum effects due to the massive scalar field.

The paper is organized as follows. In the next section, we determine the master equation for the massive, nonminimally coupled, scalar field. In Sec. III, we set out the cosmological background containing a sudden singularity, and in Sec. IV, the master equation is solved. The Bogoliubov coefficients are determined in Sec. V. In Sec. VI, we regularize the expression for the energy density, and show that the final result is zero. In Sec. VII, we discuss our conclusions.

\section{THE MASTER EQUATION}

We shall investigate quantum creation of massive particles near a sudden cosmological singularity at finite time. We aim to determine if these quantum effects are strong enough to change the evolution of the Universe near a classical sudden singularity. In order to do this, we must construct a specific model. We will concentrate on the evolution of a massive scalar field, $\phi$, nonminimally coupled to gravity, described by the following Lagrangian:

$$
\mathcal{L}=\frac{1}{2} \phi_{; \rho} \phi^{; \rho}-\frac{1}{2} m^{2} \phi^{2}+\frac{1}{12} R \phi^{2} .
$$

The presence of mass takes us one step further than previous work concerning particle production near sudden singularity [13], which considered only massless scalar particle production. The nonminimal coupling should strengthen any quantum effects near the singularity, and also introduces some technical features that facilitate obtaining exact solutions to the problem.

By variation of the Lagrangian with respect to $\phi$, we obtain the field equation

$$
\square \phi+m^{2} \phi-\frac{R}{6} \phi=0 .
$$

Under variation with respect to the metric, the Lagrangian also gives the momentum-energy tensor

$$
\begin{aligned}
T_{\mu \nu}= & \frac{2}{3} \phi_{; \mu} \phi_{; \nu}-\frac{1}{6} g_{\mu \nu} \phi_{; \rho} \phi^{; \rho}+\frac{1}{2} m^{2} g_{\mu \nu} \phi^{2} \\
& -\frac{\phi}{3}\left(\phi_{; \mu ; \nu}-g_{\mu \nu} \square \phi\right)+\frac{1}{6} G_{\mu \nu} \phi^{2},
\end{aligned}
$$

where

$$
G_{\mu \nu}=R_{\mu \nu}-\frac{1}{2} g_{\mu \nu} R
$$

is the Einstein tensor.

If we use Eq. (2) together with the flat FriedmannLemaître-Robertson-Walker (FLRW) metric for the Universe,

$$
d s^{2}=d t^{2}-a^{2}(t)\left(d x^{2}+d y^{2}+d z^{2}\right)
$$

where $a(t)$ is the expansion scale factor, we find

$$
\ddot{\phi}+3 \frac{\dot{a}}{a} \dot{\phi}+\left\{\frac{k^{2}}{a^{2}}+m^{2}+\left[\frac{\ddot{a}}{a}+\left(\frac{\dot{a}}{a}\right)^{2}\right]\right\} \phi=0,
$$

where over-dots denote derivatives with respect to the comoving proper time, $t$. This equation can be rewritten in terms of the conformal time, $\eta$, defined by $d t=a d \eta$, as

$$
\phi^{\prime \prime}+2 \frac{a^{\prime}}{a} \phi^{\prime}+\left(k^{2}+m a^{2}+\frac{a^{\prime \prime}}{a}\right) \phi=0 .
$$

The primes denote derivatives with respect to the conformal time.

Redefining the scalar field as

$$
\phi=\frac{\chi}{a},
$$

we obtain the following equation for $\chi$ :

$$
\chi^{\prime \prime}+\left(k^{2}+m^{2} a^{2}\right) \chi=0 .
$$

This is the principal equation we will work with from now on.

\section{TWO COSMOLOGICAL ERAS}

The sudden singularity can be described by the following expression for the expansion scale factor [15-17]:

$$
a(t)=\left(\frac{t}{t_{s}}\right)^{q}\left(a_{s}-1\right)+1-\left(1-\frac{t}{t_{s}}\right)^{n},
$$

where $t_{s}$ is the time where the sudden singularity occurs, and $a_{s}$ is the value of the scale factor at this moment. Moreover, $0<q \leq 1$ and $1<n<2$ where $q$ and $n$ are free constants and no relation is assumed between the pressure $p$ and the density $\rho$. We have two asymptotic phases:

(i) Primordial phase, $t \rightarrow 0$ :

$$
\begin{gathered}
a \rightarrow\left(\frac{t}{t_{s}}\right)^{q}\left(a_{s}-1\right), \\
\dot{a} \rightarrow \frac{q}{t_{s}}\left(\frac{t}{t_{s}}\right)^{q-1}\left(a_{s}-1\right), \\
\ddot{a} \rightarrow \frac{q}{t_{s}^{2}}(q-1)\left(\frac{t}{t_{s}}\right)^{q-2}\left(a_{s}-1\right) .
\end{gathered}
$$

(ii) Singular phase, $t \rightarrow t_{s}$ :

$$
\begin{gathered}
a \rightarrow a_{s}, \\
\dot{a} \rightarrow \frac{q}{t_{s}}\left(a_{s}-1\right), \\
\ddot{a} \rightarrow-\frac{n}{t_{s}^{2}}(n-1)\left(1-\frac{t}{t_{s}}\right)^{n-2} .
\end{gathered}
$$

There is a radiation-dominated primordial phase if $q=$ $1 / 2$. On the other hand, in the singular phase, the scale factor and its first derivative approach constants, and the second derivative, $\ddot{a}$, diverges as $t \rightarrow t_{s}$, since $n<2$. 
In terms of the conformal time, $d \eta=a^{-1} d t$, we have for the scale factor evolution to leading order:

(i) Radiation phase:

$$
a=a_{0} \eta
$$

(ii) Singular phase:

$$
a=a_{s} .
$$

The scale factor and its first derivative must be continuous during the transition of one phase to another. If $a_{s}$ is the scale factor value at the moment of the transition, and $H_{0}$ the corresponding Hubble parameter, then the transition moment is given by $\eta_{t}=1 /\left(H_{0} a_{s}\right)$ and $a_{0}=H_{0} a_{s}^{2}$.

The isotropic and homogeneous form we have assumed for the cosmological evolution of the scale factor, $a(t)$, towards a sudden singularity captures the essential features of the general solution near such a singularity. On approach to the sudden singularity as $t \rightarrow t_{s}$, the FLRW solution has the linear asymptotic form

$$
a \rightarrow a_{s}+q\left(1-a_{s}\right)\left(1-\frac{t}{t_{s}}\right) .
$$
form

We can generalize it to an inhomogeneous metric of the

$$
d s^{2}=d t^{2}-\left(a_{\alpha \beta}+\tau b_{\alpha \beta}+\tau^{n} c_{\alpha \beta}+\ldots\right) d x^{\alpha} d x^{\beta}
$$

on approach to a sudden singularity at $\tau \equiv t-t_{s}=0$, where $a_{\alpha \beta}, b_{\alpha \beta}$ and $c_{\alpha \beta}$ with $\alpha, \beta=1,2,3$ are functions of the space coordinates and $n$ is a constant such that $1<n<2$. In the absence of an equation of state, nine components of the symmetric $a_{\alpha \beta}, b_{\alpha \beta}$ and $c_{\alpha \beta}$ tensors are left independent and arbitrary by the field equations as $\tau \rightarrow 0$ and so (14) is characteristic of part of the general solution of the Einstein equations in the vicinity of a sudden singularity [18]. A stability analysis of the FLRW solution has also been performed by Barrow and Lip [19]. For other studies of sudden singularities of this type, in general relativity and related theories of gravity, see Refs. [20-32].

\section{THE SOLUTIONS FOR THE MASTER EQUATIONS}

Let us return to the master equation (4). First, note that if the mass is zero, $m=0$, then during both phases the equation becomes

$$
\chi^{\prime \prime}+k^{2} \chi=0 .
$$

The solution is the same during these two phases, and they can be written in the form of plane waves. Since the solution must be continuous, there is no final effect in the singular phase, for the trace anomaly or for particle production.
If the mass is nonzero, the equation during the singular phase takes the form

$$
\chi^{\prime \prime}+\left(k^{2}+m^{2} a_{s}^{2}\right) \chi=0 .
$$

The solution is still of plane wave form, but with a frequency that is affected by the presence of the mass term. During the radiative phase, the equation is

$$
\chi^{\prime \prime}+\left(k^{2}+m^{2} a_{0}^{2} \eta^{2}\right) \chi=0
$$

which can be rewritten as

$$
\ddot{\chi}+\left(\omega+\frac{x^{2}}{4}\right) \chi=0,
$$

where $x=\sqrt{2 m a_{0}} \eta, \omega=k^{2} / 2 m a_{0}$, with dots now denoting derivatives with respect to $x$. This is a parabolic cylinder equation, and the solutions can be written as

$$
\chi=e^{-i\left(x^{2} / 4\right)}\left[c_{11} F_{1}\left(r, s, i \frac{x^{2}}{2}\right)+c_{2} x_{1} F_{1}\left(p, q, i \frac{x^{2}}{2}\right)\right],
$$

where the ${ }_{1} F_{1}$ are confluent hypergeometric functions (Kummer functions) [33], with

$$
\begin{array}{ll}
r=i \frac{\omega}{2}+\frac{1}{4}, & s=\frac{1}{2}, \\
p=i \frac{\omega}{2}+\frac{3}{4}, & s=\frac{3}{2},
\end{array}
$$

where $c_{1,2}$ are constants.

The confluent hypergeometric functions may be expressed as a series:

$$
{ }_{1} F_{1}(r, s, z)=1+\frac{r}{s} z+\frac{1}{2} \frac{r(r+1)}{s(s+1)} z^{2}+\ldots
$$

The series represented by the constant $c_{1}$ is even, while the solution represented by the constant $c_{2}$ is odd.

Let us rewrite the solution as

$$
\chi=c_{1} \chi_{1}+c_{2} \chi_{2} .
$$

Considering the series expansion described above, we find

$$
\begin{aligned}
& \chi_{1}=1-\omega \frac{x^{2}}{2}-\frac{1}{4}\left(\frac{1}{12}-\frac{\omega^{2}}{6}\right) x^{4}+\ldots \\
& \chi_{2}=x-\omega \frac{x^{3}}{6}-\frac{1}{4}\left(\frac{1}{20}-\frac{\omega^{2}}{30}\right) x^{5}+\ldots
\end{aligned}
$$

An important detail is that if we want to take into account the mass in Eq. (17) we must consider the series at least until order $x^{5}$ ( $x^{4}$ for the even series), while if the mass is not taken into account we can stop at order $x^{3}$ ( $x^{2}$ for the even series). The initial conditions are imposed for $\eta \rightarrow 0$ $(x \rightarrow 0)$ when the mass is negligible. Considering the expansion for the massless case, and going back to the notation in terms of the conformal time, we find 


$$
\begin{gathered}
\chi=c_{1} \chi_{1}+c_{2} \chi_{2} \sim c_{1}\left(1-\frac{k^{2} \eta^{2}}{2}\right)+c_{2} \frac{\sqrt{2 m a_{0}}}{k}\left(k \eta-\frac{k^{3} \eta^{3}}{6}\right) \\
\sim c_{1} \cos k \eta+c_{2} \frac{\sqrt{2 m a_{0}}}{k} \sin k \eta .
\end{gathered}
$$

Choosing

$$
c_{1}=\frac{1}{\sqrt{2 k}}, \quad c_{2}=\frac{k}{\sqrt{2 m a_{0}}} \frac{i}{\sqrt{k}},
$$

the solution for $\eta \rightarrow 0$ can be written as

$$
\chi \sim \frac{1}{\sqrt{2 k}} e^{-i k \eta},
$$

corresponding to the vacuum initial state.

With those choices for the constants $c_{1}$ and $c_{2}$, we now have the solution at any time, also for the massive case

$$
\chi=\frac{1}{\sqrt{2 k}} e^{-i\left(x^{2} / 4\right)}\left[{ }_{1} F_{1}\left(r, s, i \frac{x^{2}}{2}\right)+i \sqrt{a} x_{1} F_{1}\left(p, q, i \frac{x^{2}}{2}\right)\right],
$$

where we have restored the mass in the expressions.

\section{THE BOGOLIUBOV COEFFICIENTS}

Suppose that we approximate the solution during the radiation era by a massless field. This is equivalent to assuming that we have the massive solution shown above, but in the limit $\eta \rightarrow 0$. Hence, we have the following solutions corresponding to the two phases:

$$
\begin{gathered}
\phi_{k}(\eta)=\frac{e^{i k \eta}}{\sqrt{2 k}} \quad \text { (primordial phase), } \\
\phi_{k}(\eta)=\xi_{01} e^{i \tilde{\omega} \eta}+\xi_{02} e^{-i \tilde{\omega} \eta} \quad \text { (singular phase), }
\end{gathered}
$$

where $\xi_{01,02}$ are constants and $\tilde{\omega}=\sqrt{k^{2}+m^{2} a_{0}^{2}}$.

Now we impose the matching conditions for these two solutions at $\eta=\eta_{c}$ by requiring continuity of the function and of its first derivative. We obtain the following relations:

$$
\begin{aligned}
& \xi_{01}=\frac{1}{2} \frac{1}{\sqrt{2 k}}\left(1+\frac{k}{\tilde{\omega}}\right) e^{i(k-\tilde{\omega}) \eta_{c},} \\
& \xi_{02}=\frac{1}{2} \frac{1}{\sqrt{2 k}}\left(1-\frac{k}{\tilde{\omega}}\right) e^{i(k+\tilde{\omega}) \eta_{c}} .
\end{aligned}
$$

We notice that when $m=0, \xi_{01}=1 / \sqrt{2 k}$ and $\xi_{02}=0$ and so the solution is the same in the two phases and there are no particle production effects.

On imposing the quantization, we find the following expressions for the two phases:

$$
\phi_{k}(\eta)=\frac{e^{i k \eta}}{\sqrt{2 k}} a+\frac{e^{-i k \eta}}{\sqrt{2 k}} a^{\dagger} \quad \text { (primordial phase), }
$$

$\phi_{k}(\eta)=\left(\xi_{01} e^{i \tilde{\omega} \eta}+\xi_{02} e^{-i \tilde{\omega} \eta}\right) a+\left(\xi_{01}^{*} e^{-i \tilde{\omega} \eta}+\xi_{02}^{*} e^{i \tilde{\omega} \eta}\right) a^{\dagger}$

(singular phase),

where $a$ and $a^{\dagger}$ are the creation and annihilation operators. These solutions (and their derivatives) are continuous at $\eta=\eta_{c}$. They can be rewritten as

$$
\begin{aligned}
\phi_{k}(\eta) & =\frac{e^{i k \eta}}{\sqrt{2 k}} a+\frac{e^{-i k \eta}}{\sqrt{2 k}} a^{\dagger} \quad \text { (primordial phase), } \\
\phi_{k}(\eta) & =\frac{e^{i \tilde{\omega} \eta}}{\sqrt{2 \tilde{\omega}}} b+\frac{e^{-i \tilde{\omega} \eta}}{\sqrt{2 \tilde{\omega}}} b^{\dagger} \quad \text { (singular phase). }
\end{aligned}
$$

Hence, we have

$$
b=\sqrt{2 \tilde{\omega}}\left(\xi_{01} a+\xi_{02} a^{\dagger}\right) .
$$

In this way, we find expressions for the Bogoliubov coefficients that connect the quantum modes during the different phases [34]:

$$
\alpha=\sqrt{2 \tilde{\omega}} \xi_{01}, \quad \beta=\sqrt{2 \tilde{\omega}} \xi_{02} .
$$

The normalization condition,

$$
\alpha \alpha^{*}-\beta \beta^{*}=1,
$$

is satisfied. When the mass is zero (i.e., a conformally coupled scalar field), we have $\alpha=1$ and $\beta=0$. The coefficient $\beta$ is associated with the created particles [34]. Hence, the number of created particles for each mode $k$ is

$$
N_{k}=\beta \beta^{*}=\frac{1}{4}\left(1-\frac{k}{\tilde{\omega}}\right)^{2},
$$

while the energy of each mode is

$$
\rho_{k}=k N_{k} \text {. }
$$

An integration over all $k$-modes gives

$$
\rho=\int_{0}^{\infty} \rho_{k} d^{3} k=\pi \int_{0}^{\infty} k^{2} \tilde{\omega}\left(1-\frac{k}{\tilde{\omega}}\right)^{2} d k .
$$

This expression clearly diverges so it is necessary to regularize it. But, heuristically, since it is a polynomial expression, it seems clear that after regularization we must obtain zero. Hence, the particle production should not contribute to the energy-momentum tensor and the sudden singularity is unaffected by these quantum effects.

Note that the integral (40) admits an analytical solution:

$$
\begin{aligned}
\int \rho_{k} d^{3} k= & \pi \int k^{2} \tilde{\omega}\left(1-\frac{k}{\tilde{\omega}}\right)^{2} d k \\
= & \pi\left\{k \sqrt{k^{2}+\bar{m}^{2}}\left(\frac{k^{2}}{2}-\frac{\bar{m}^{2}}{4}\right)-\frac{k^{4}}{2}\right. \\
& \left.+\frac{\bar{m}^{2}}{4} \ln \left[2\left(k+\sqrt{k^{2}+\bar{m}}\right)\right]\right\},
\end{aligned}
$$

with $\bar{m}=m a_{0}$. There is no infrared divergence, but there is a logarithmic divergence when $k \rightarrow \infty$ (ultraviolet limit). 


\section{REGULARIZING THE ENERGY}

In order to regularize the expression of the energy, we use the $n$-wave method expounded in Ref. [35]. This method is based on the Pauli-Villars technique used for quantum field theory in Minkowski spacetime. First, let us write the energy as

$$
\rho=\int_{0}^{\infty} \rho_{k}(k, m) k^{2} d k
$$

Let us define

$$
\rho_{k}^{(n)} \equiv \frac{1}{n} \rho_{k}(n k, n m)
$$

where $n$ is a parameter that characterizes the order of the divergence. From this expression, we construct the quantities

$$
E_{k}^{p}=\lim _{n \rightarrow \infty} \frac{\partial^{p} \rho_{k}^{(n)}}{\partial\left(n^{-2}\right)^{p}}
$$

The expression for the regularized energy is given by

$$
\rho_{k}^{\mathrm{reg}}=\rho_{k}-E_{k}^{0}-E_{k}^{1}-\frac{1}{2} E_{k}^{2},
$$

where $E_{k}^{0}$ eliminates the logarithmic divergence, $E_{k}^{1}$ the quadratic divergence, and $E_{k}^{2}$ the quartic divergence-all those that are normally present in the energy-momentum tensor. This regularization of the energy corresponds to a full renormalization of the coupling constants, as described in $[36,37]$.

We have,

$$
\rho_{k}=\sqrt{k^{2}+\bar{m}^{2}}-2 k+\frac{k^{2}}{\sqrt{k^{2}+\bar{m}^{2}}} .
$$

It follows that

$$
\rho_{k}^{(n)}=\rho_{k} .
$$

Hence, only the zero-order term survives, and leads to

$$
\rho_{k}^{\mathrm{ren}}=\rho_{k}-E_{k}^{0}=\rho_{k}-\rho_{k}=0 .
$$

As we suspect, the renormalized energy is zero. There is no effect, and the quantum phenomena associated with the cosmological dynamics do not change the character of the sudden singularity or prevent its occurrence.

\section{CONCLUSIONS}

In this work, we have investigated the fate of the universe near a future sudden singularity due to quantum particle production effects by a massive scalar field that is nonminimally coupled to gravity. We have used the method of calculating Bogoliubov coefficients and the expression obtained for the energy of the created particles near the singularity is divergent. This divergence is cured using the standard $n$-wave method and the final result after regularization is exactly zero. Hence, we can conclude that the sudden singularity is robust against quantum effects due to the presence of a massive scalar field. We have used a simple description for the background cosmological evolution toward a sudden singularity at finite time that shares the same time evolution as part of the general solution of the Einstein equations near such a singularity.

An interesting consequence of this result concerns the trace anomaly. A massless scalar field conformally coupled to gravity is a particular case of the problem studied here. For this particular case the result is the same, with no quantum effects. Hence, the trace anomaly is absent near the singularity, as had been speculated in Ref. [13]. It must also be stressed, concerning this massless limit, that the same regularization method can be used since there is no infrared divergence even when the mass is zero.

Other fields should be considered to test the robustness of a classical sudden singularity with respect to quantum effects. However, since the scalar field and its first derivative are both constant at the sudden singularity, the KleinGordon equation implies that in general there will only be a change in the frequency of the quantum modes as the sudden singularity is approached. This frequency change alone is unable to create influential quantum effects. Hence, we can surmise that perhaps the result found here and in Refs. $[13,14]$ can be generalized further, and they can be applied to other types of fields.

One such a generalization arises if we consider a nonconformal coupling, with a Klein-Gordon equation given by

$$
\square \phi+m^{2} \phi-\xi R \phi=0
$$

For a flat FLRW metric, this equation reduces to

$$
\phi^{\prime \prime}+2 \frac{a^{\prime}}{a} \phi^{\prime}+\left\{k^{2}+m^{2} a^{2}+6 \xi \frac{a^{\prime \prime}}{a}\right\} \phi=0 .
$$

For the radiative phase, the scenario is the same as that studied above, since $a^{\prime \prime}=0$. For the sudden singularity phase, the situation is more involved since there is a singularity in $a^{\prime \prime}$. However, a transformation of the type $\phi=$ $a^{-6 \xi} \chi$ (which reduces to our previous transformation for $\xi=6$ - the conformal coupling) can eliminate this singularity, leading to a regular equation with a damped (antidamped) harmonic oscillator equation for $\xi>\frac{1}{6}\left(\xi<\frac{1}{6}\right)$. This new term seems to be harmless since the dissipation (antidissipation) effect lasts only for a finite time until the singularity is reached. Hence, we expect that our previous results hold even in this more general case. 
Another way to consider the problem of quantum avoidance of future singularities is to solve to Wheeler-de Witt equation in universes containing future singularities. For big rip and big brake singularities, this question has been analyzed in Refs. [38,39], with some indications that, at least for the big brake case, the singularity can be avoided due to quantum effects. For the big rip case the situation is less clear, see also Refs. [40,41]. It is a natural step to perform such an analysis for the sudden singularity case, an issue we hope to consider in the future.

\section{ACKNOWLEDGMENTS}

We thank CNPq (Brasil) for partial financial support.
[1] E. Komatsu et al., Astrophys. J. Suppl. Ser. 192, 18 (2011).

[2] R. R. Caldwell, Phys. Lett. B 545, 23 (2002).

[3] J. D. Barrow, G. J. Galloway, and F. J. Tipler, Mon. Not. R. Astron. Soc. 223, 835 (1986).

[4] A. A. Starobinsky, Gravitation Cosmol. 6, 157 (2000).

[5] V. Gorini, A. Y. Kamenshchik, U. Moschella, and V. Pasquier, Phys. Rev. D 69, 123512 (2004).

[6] Z. Keresztes, L. Á. Gergely, V. Gorini, U. Moschella, and A. Yu. Kamenshchik, Phys. Rev. D 79, 083504 (2009).

[7] Z. Keresztes, L. A. Gergely, A. Y. Kamenshchik, V. Gorini, and D. Polarski, Phys. Rev. D 82, 123534 (2010).

[8] F. J. Tipler, Phys. Lett. A 64, 8 (1977).

[9] A. Królak, Classical Quantum Gravity 3, 267 (1986).

[10] A. B. Batista, J.C. Fabris, and S. Houndjo, Gravitation Cosmol. 14, 140 (2008).

[11] F. G. Alvarenga, A. B. Batista, J.C. Fabris, and S. Houndjo, Gravitation Cosmol. 16, 105 (2010).

[12] J. D. Bates and P. R. Anderson, Phys. Rev. D 82, 024018 (2010).

[13] J. D. Barrow, A. B. Batista, J. C. Fabris, and S. Houndjo, Phys. Rev. D 78, 123508 (2008).

[14] S. J. M. Houndjo, Europhys. Lett. 92, 10004 (2010).

[15] J. D. Barrow, Classical Quantum Gravity 21, L79 (2004).

[16] J. D. Barrow, Classical Quantum Gravity 21, 5619 (2004).

[17] J. D. Barrow and C. G. Tsagas, Classical Quantum Gravity 22, 1563 (2005).

[18] J.D. Barrow, S. Cotsakis, and A. Tsokaros, Classical Quantum Gravity 27, 165017 (2010).

[19] J. D. Barrow and S.Z.W. Lip, Phys. Rev. D 80, 043518, (2009).

[20] H. Stefancic, Phys. Rev. D 71, 084024 (2005).

[21] E. J. Copeland, M. Sami, and S. Tsujikawa, Int. J. Mod. Phys. D 15, 1753 (2006).

[22] S. Cotsakis and I. Klaoudatou, J. Geom. Phys. 57, 1303 (2007).
[23] Y. Shtanov and V. Sahni, Classical Quantum Gravity 19, L101 (2002).

[24] M. P. Dạbrowski, Phys. Rev. D 71, 103505 (2005).

[25] S. Nojiri and S. D. Odintsov, arXiv:hep-th/0412030.

[26] S. Nojiri, S. D. Odintsov and S. Tsujikawa, Phys. Rev. D 71, 063004 (2005).

[27] C. Cattoen and M. Visser, Classical Quantum Gravity 22, 4913 (2005).

[28] M. P. Da̧browski, Phys. Lett. B 625, 184 (2005).

[29] M. P. Da̧browski, T. Denkiewicz, and M. A. Hendry, Phys. Rev. D 75, 123524 (2007).

[30] K. Lake, Classical Quantum Gravity 21, L129 (2004).

[31] L. Fernandez-Jambrina and R. Lazkoz, Phys. Rev. D 70, 121503R (2004).

[32] L. Fernandez-Jambrina and R. Lazkoz, Phys. Rev. D 74, 064030 (2006).

[33] M. Abramowitz and I. A. Stegun, Handbook of Mathematical Functions (Dover, New York, 1970), p. 508.

[34] N.D. Birrell and P.C.W. Davies, Quantum Fields in Curved Space (Cambridge University Press, Cambridge, 1982).

[35] Ya. B. Zel'dovich and A. A. Starobinsky, Sov. Phys. JETP 34, 1159 (1972).

[36] A. A. Grib, S. G. Mamayev, and V. M. Mostepanenko, Vacuum Quntum Effects in Strong Fields (Friedmann, St. Petersburg, 1994).

[37] M. Bordag, J. Lindig, and V. M. Mostepanenko, Classical Quantum Gravity 15, 581 (1998).

[38] M. P. Dabrowski, C. Kiefer, and B. Sandhofer, Phys. Rev. D 74, 044022 (2006).

[39] A. Kamenshchik, C. Kiefer, and B. Sandhofer, Phys. Rev. D 76, 064032 (2007).

[40] E. M. Barboza Jr. and N. A. Lemos, Gen. Relativ. Gravit. 38, 1609 (2006).

[41] N. Pinto-Neto and D. M. Pantoja, Phys. Rev. D 80, 083509 (2009). 